\title{
Piezoelectric Wafer Active Sensors for Structural Health Monitoring of Composite Structures Using Tuned Guided Waves
}

\section{Victor Giurgiutiu \\ University of South Carolina, Columbia, SC 29208}

\begin{abstract}
Piezoelectric wafer active sensors (PWAS) are lightweight and inexpensive transducers that enable a large class of structural health monitoring (SHM) applications such as: (a) embedded guided wave ultrasonics, i.e., pitch-catch, pulse-echo, phased arrays; (b) highfrequency modal sensing, i.e., the electro-mechanical $(E / M)$ impedance method; and $(c)$ passive detection (acoustic emission and impact detection). The focus of this paper is on the challenges posed by using PWAS transducers in the composite structures as different from the metallic structures on which this methodology was initially developed. After a brief introduction, the paper reviews the PWAS-based SHM principles. It follows with a discussion of guided wave propagation in composites and PWAS tuning effects. Then, it discusses damage modes in composites. Finally, the paper presents some experimental results with damage detection in composite specimens. Hole damage and impact damage were detected using pitch-catch method with tuned guided waves being sent between a transmitter PWAS and a received PWAS. Root mean square deviation (RMSD) damage index (DI) were shown to correlate well with hole size and impact intensity. The paper ends with summary and conclusion; suggestions for further work are also presented. [DOI: $10.1115 / 1.4004698]$
\end{abstract}

Keywords: composites, composite structures, structural integrity, structural health monitoring, piezoelectric wafer active sensors, SHM, NDE, PWAS

\section{Introduction}

Structural health monitoring (SHM) is an emerging technology with multiple applications in the evaluation of critical structures. The goal of SHM research is to develop a monitoring methodology that is capable of detecting and identifying, with minimal human intervention, various damage types during the service life of the structure. Numerous approaches have been utilized in recent years to perform structural health monitoring [1-3]; they can be broadly classified into two categories: passive methods and active methods. Passive SHM methods (such as acoustic emission, impact detection, strain measurement, etc.) have been studied longer and are relatively mature; however, they suffer from several drawbacks which limit their utility (need for continuous monitoring, indirect inference of damage existence, etc.). Active SHM methods are currently of greater interest due to their ability to perform on-demand interrogation of a structure while the structure is still in service. One of the promising active SHM methods utilizes arrays of piezoelectric wafer active sensors (PWAS) bonded to a structure for both transmitting and receiving ultrasonic waves in order to achieve damage detection [4]. When used to interrogate thin-wall structures, the PWAS are effective guided wave transducers which couple their in-plane motion with the guided wave particle motion on the material surface. The in-plane PWAS motion is excited by an applied high-frequency voltage through the $d_{31}$ piezoelectric coupling. Optimum excitation and detection takes place when the PWAS length is in certain ratios with the wavelength of the guided wave modes. The PWAS action as ultrasonic transducers is fundamentally different from that of conventional ultrasonic transducers. Conventional ultrasonic transducers

Contributed by the Materials Division of ASME for publication in the Journal OF Engineering Materials and Technology. Manuscript received March 13, 2011; final manuscript received July 13, 2011; published online October 20, 2011. Assoc. Editor: Mrinal Saha. act through surface tapping, i.e., by applying vibration pressure to the structural surface. The PWAS transducers act through surface pinching and are strain coupled with the structural surface. This allows the PWAS transducers to have a greater efficiency in transmitting and receiving ultrasonic surface and guided waves when compared with the conventional ultrasonic transducers.

There has been a marked increase in recent years in the use of composite materials in numerous types of structures. Composites have gained popularity in high-performance products that need to be lightweight, yet strong enough to take high loads such as aerospace structures, space launchers, satellites, and racing cars. Their growing use has arisen from their high specific strength and stiffness in comparison with metals and from the ability to shape and tailor a structure to produce more efficient configurations [5].

For this reason, it is important to study how active SHM methods (which were initially developed for isotropic metallic structures) can be extended to detecting damage in composite materials, which are multilayered and anisotropic. For example, one of the most troubling forms of damage in laminated composites is low-velocity impact damage. This type of damage can leave no visual traces, but subsurface delaminations can significantly reduce the strength of the structure. Finding this type of damage is very challenging.

The present paper presents and discusses the challenges and opportunities related to the use of PWAS in generating and sensing ultrasonic guided waves in composite materials and how they can be used to detect damage in composite structures. The paper starts with a review of the general principles of PWAS-based SHM. Then, it discusses the analytical challenges of studying guided waves in composites and shows how the concept of guided wave tuning with PWAS can be applied in the case of composite structures: theoretical predictions and experimental results of PWAS-guided waves tuning in a composite plate are comparatively presented. Subsequently, the paper gives a brief presentation of the main composite materials damage types which are 


\section{Propagating Lamb waves}
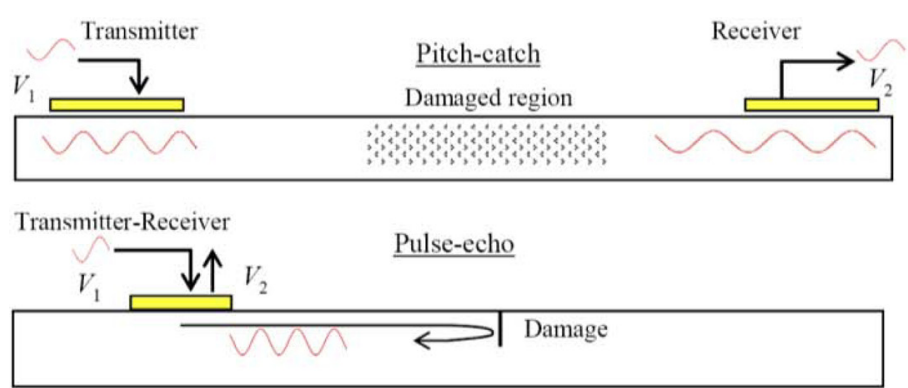

Transmitter-Receiver
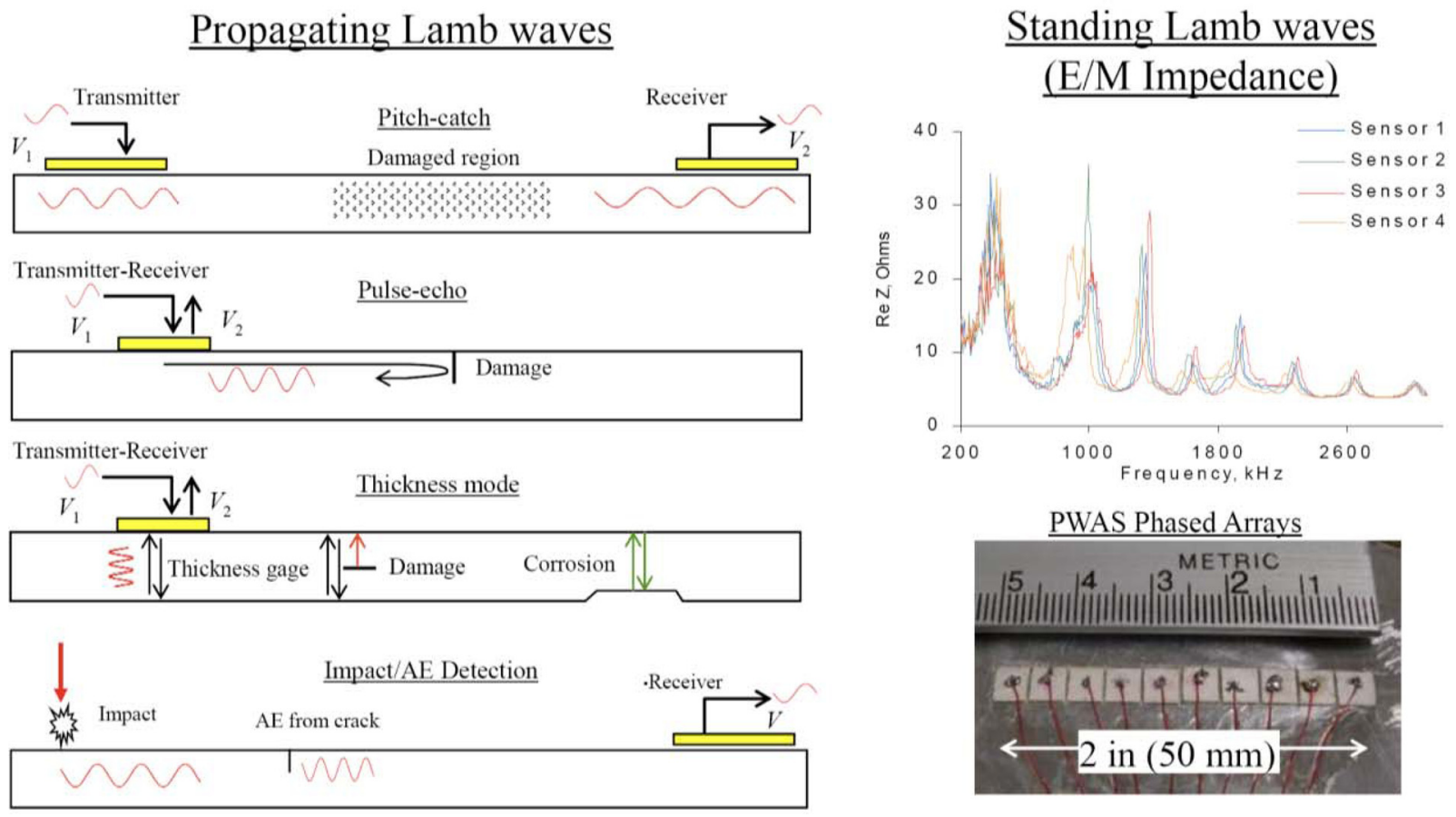

Fig. 1 Modes of operation of piezoelectric wafer active sensors (PWAS) transducers: (a) propagating guided Lamb waves; (b) standing guided Lamb waves; (c) PWAS phased arrays

often different from those encountered in metallic structures. Finally, the paper presents experimental results on detecting hole and impact damage in composite plates with PWAS-based active SHM methods. The final part of the paper presents the conclusions and makes suggestions for further work.

\section{PWAS Principles}

PWAS are the enabling technology for active SHM systems. PWAS couples the electrical and mechanical effects (mechanical strain, $S_{i j}$, mechanical stress, $T_{k l}$, electrical field, $E_{k}$, and electrical displacement $D_{j}$ ) through the tensorial piezoelectric constitutive equations

$$
\begin{aligned}
& S_{i j}=s_{i j k l}^{E} T_{k l}+d_{k i j} E_{k} \\
& D_{j}=d_{j k l} T_{k l}+\varepsilon_{j k}^{T} E_{k}
\end{aligned}
$$

where, $s_{i j k l}^{E}$ is the mechanical compliance of the material measured at zero electric field $(E=0), \varepsilon_{j k}^{T}$ is the dielectric permittivity measured at zero mechanical stress $(T=0)$, and $d_{k i j}$ represents the piezoelectric coupling effect. PWAS utilize the $d_{31}$ coupling between in-plane strain and transverse electric field. A 7-mm diameter PWAS, $0.2 \mathrm{~mm}$ thin, weighs a bare $78 \mathrm{mg}$ and costs around $\sim \$ 1$ each. PWAS are lightweight and inexpensive and hence can be deployed in large numbers on the monitored structure. Just like conventional ultrasonic transducers, PWAS utilize the piezoelectric effect to generate and receive ultrasonic waves. However, PWAS are different from conventional ultrasonic transducers in several aspects:

1. PWAS are firmly coupled with the structure through an adhesive bonding, whereas conventional ultrasonic transducers are weakly coupled through gel, water, or air.

2. PWAS are nonresonant devices that can be tuned selectively into several guided wave modes, whereas conventional ultrasonic transducers are resonant narrow-band devices.

3. PWAS are inexpensive and can be deployed in large quantities on the structure, whereas conventional ultrasonic transducers are expensive and used one at a time.

By using Lamb waves in a thin-wall structure, one can detect structural anomaly, i.e., cracks, corrosions, delaminations, and other damage. Because of the physical, mechanical, and piezoelectric properties of PWAS transducers, they act as both transmitters and receivers of Lamb waves traveling through the structure. Upon excitation with an electric signal, the PWAS generate Lamb waves in a thin-wall structure. The generated Lamb waves travel through the structure and are reflected or diffracted by the structural boundaries, discontinuities, and damage. The reflected or diffracted waves arrive at the PWAS where they are transformed into electric signals.

PWAS transducers can serve several purposes [4,6-8]: (a) highbandwidth strain sensors; (b) high-bandwidth wave exciters and receivers; (c) resonators; (d) embedded modal sensors with the electromechanical (E/M) impedance method. The PWAS transducers have various modes of operation (Fig. 1): (i) active sensing of far-field damage using pulse-echo, pitch-catch, and phased array methods, (ii) active sensing of near-field damage using highfrequency $\mathrm{E} / \mathrm{M}$ impedance method and thickness-gage mode, and (iii) passive sensing of damage-generating events through detection of low-velocity impacts and acoustic emission at the tip of advancing cracks. Damage detection using PWAS phased arrays can detect both broadside and offside cracks independently with scanning beams emitting from a central location.

\section{Guided Waves in Composites}

Composite materials combine the properties of two or more constituent materials in order to achieve properties that are not achievable by the individual constituents. For example, carbon fiber reinforced plastics (CFRP) composites combine the extreme specific stiffness and strength of carbon fibers with the binding properties of a polymeric matrix. For high-performance structural applications, laminated composites made up of high strength/ stiffness unidirectional layers stacked at various angles have gained wide application. The choice of the orientation angles in the stacking sequence allows tailoring of the composite properties along certain preferential directions that are expected to experience highest operational loads. The simplest layup sequence is the $0 / 90$ (cross-ply) laminate, which consists of alternating 0-deg and 90-deg layers. Some layup sequences such as 0/45/90 and $0 / 60 / 120$ are dubbed "quasi-isotropic" because they try to 

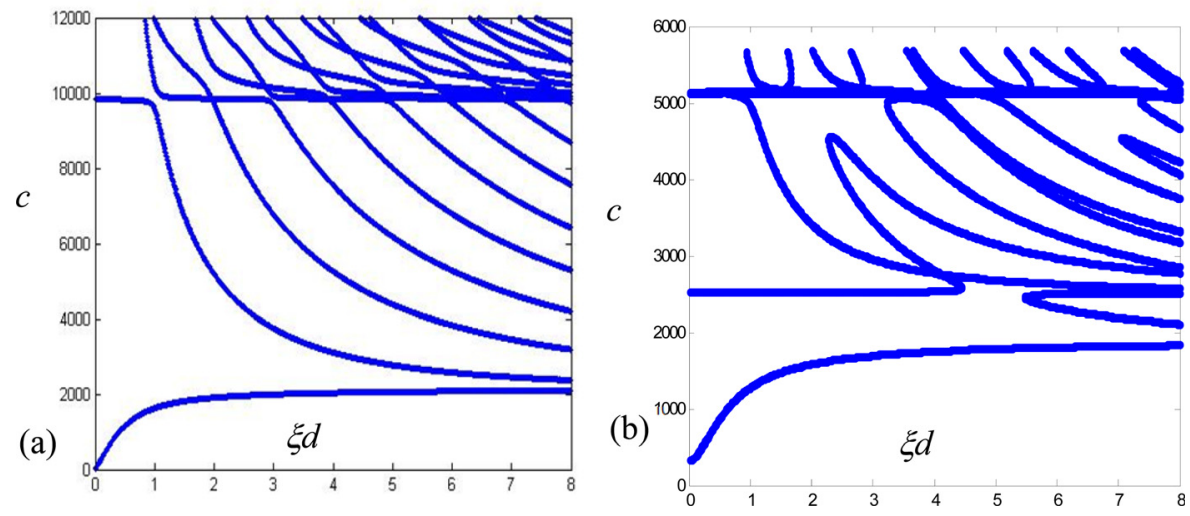

Fig. 2 Dispersion curves for unidirectional 65/35 graphite-epoxy plate: (a) $\theta=0^{\circ}$; $(b)$ $\theta=36^{\circ}$. Note: $c$ is the wave speed in $\mathrm{m} / \mathrm{s} ; \xi d$ is the dimensionless wavenumber-half thickness product.

equalize the effective properties by applying the fibers in several directions.

The evaluation of structural integrity using guided Lamb wave ultrasonics has long been acknowledged as a very promising technique. Several investigators [9-11] have envisioned the inspection of large metallic plates from a single location using transducer arrays where each element acts as both transmitter and receiver. Guided signals are generated at different angles around the transducer positions and the signal reflections from the boundaries are processed for damage detection. This configuration is very promising for isotropic material but might have some limitations for fibrous composite structures due to the change in properties with fiber direction. In recent years, numerous investigations have explored Lamb wave techniques for the detection of damage in composite laminates [12]. In order to take full advantage of Lamb wave techniques for composite damage detection, one needs to first understand and model how guided waves propagate in composite structures which are much more complicated than in isotropic metallic structures.

The guided waves propagating in composite structures are more difficult to model than those propagating in isotropic metallic structures because of the composite material is inherent anisotropy and multilayered, with each layer having a different orientation. A composite plate is made of $N$ layers; each layer is made of unidirectional fibers, which give the layer in orthotropic properties; the fiber orientation varies from layer to layer according to the layup sequence. The study of wave propagation in a laminated composite is achieved by linking together the wave propagation in each composite layer with that in adjacent layers using the equilibrium and compatibility conditions at the interfaces between layers and boundary conditions at the top and bottom surfaces of the plate.

Dispersion Curves for Composite Structures. We developed a computer code to predict the wave propagation properties in a laminated composite using the analysis method presented in Ref. [13]. With this computer code, we run a number of simulation examples. First, we analyzed the dispersion curves for a unidirectional graphite-epoxy composite (65\% graphite, $35 \%$ epoxy, v/v). Figure 2 shows these dispersion curves for two orientation angles, $\theta=0^{\circ}$ and $\theta=36^{\circ}$. It is apparent that, for $\theta=0^{\circ}$ (i.e., wave propagating along the fiber direction) the dispersion curves of the symmetric, antisymmetric, and shear horizontal modes are clearly decoupled. However, for the off-axis direction $\theta=36^{\circ}$, the three mode types are strongly coupled. The wave velocity is higher when the wave propagates along the fiber direction. As the angle of the wave propagation direction increases, the phase velocity decreases till reaching a minimum in the direction perpendicular to the fiber. This is due to the fact that along the fiber the material stiffness is greater than in all the other directions and it decreases while $\theta$ increases. When we analyzed the dispersion curves for a $\left[(0 / 45 / 90 /-45)_{2 s}\right]$ quasi-isotropic CFRP laminated composite plate (upon which we later conducted experimental tests) we found even more complicated dispersion curves. However, we observed that at low $\xi d$ values a simpler pattern emerges with only the quasi-A0, quasi-S0, and quasi-SH0 modes being present.

\section{Tuned Guided Waves in Composite Structures}

The tuning between PWAS transducers and guides waves in isotropic metallic plates is relatively well understood and modeled [4]. The gist of the concept is that manipulation of PWAS size and frequency allow for selective preferential excitation of certain guided wave modes and the rejection of other guided wave modes, as needed by the particular SHM application. A similar tuning effect is also possible in anisotropic composite plates, only that the analysis is more complicated due to the anisotropic wave propagation characteristics inherent in composite materials.

To verify our analysis, we performed experiments on a 1240 $\mathrm{mm}$ by $1240-\mathrm{mm}$ quasi-isotropic composite plate with a PWAS transmitter and several PWAS receivers installed along various directions with respect to the fiber orientation in the top layer of the composite plate. The plate had an overall thickness of 2.25 $\mathrm{mm}$; the plate a $[(0 / 45 / 90 /-45) 2]_{\mathrm{S}}$ layup from T300/5208 carbon fiber unidirectional prepreg. Figure 3 shows the central part of the composite plate where 7-mm round PWAS transducers $(0.2-\mathrm{mm}$ thick, American Piezo Ceramics APC-850) were installed. The PWAS denoted with the letter $\mathrm{T}$ was the transmitter while those denoted with $\mathrm{R}$ were the receivers (R1...R5). The distance between the receivers and the transmitter was $250 \mathrm{~mm}$. The increment angle between sequential receivers was $\Delta \theta=22.5^{\circ}$. In addition, a pair of $7-\mathrm{mm}$ square PWAS were placed along the fiber direction, with $\mathrm{S} 1$ being the transmitter and S2 the receiver. Smoothed 3-count tone-burst excitation signals were used with frequency varying from $15 \mathrm{kHz}$ to $600 \mathrm{kHz}$ in steps of $15 \mathrm{kHz}$. At each frequency, we collected the wave amplitude and the time of flight for all the waves present. Three guided wave modes were detected: quasi-S0, quasi-A0, and quasi-SH0. Figure 4(a) shows the experimentally measured signal amplitudes for the three guided waves. The quasi-A0 reaches a peak response at around 50 $\mathrm{kHz}$ and then decreases. In fact, the quasi-A0 mode disappears as soon as the quasi-SH wave appears. The quasi-S0 mode reaches a peak at $450 \mathrm{kHz}$ and then decreases. The quasi-SH0 mode reaches a peak response at around $325 \mathrm{kHz}$. Figure $4(b)$ shows a comparison between theoretical prediction and experimental values for the A0 mode; the match between theory and experiment is quite good, which gives confidence in our modeling approach.

\section{Damage in Composite Materials}

The damage and failure of metallic structures is relatively well understood; their in service damage and failure occurs mostly due 


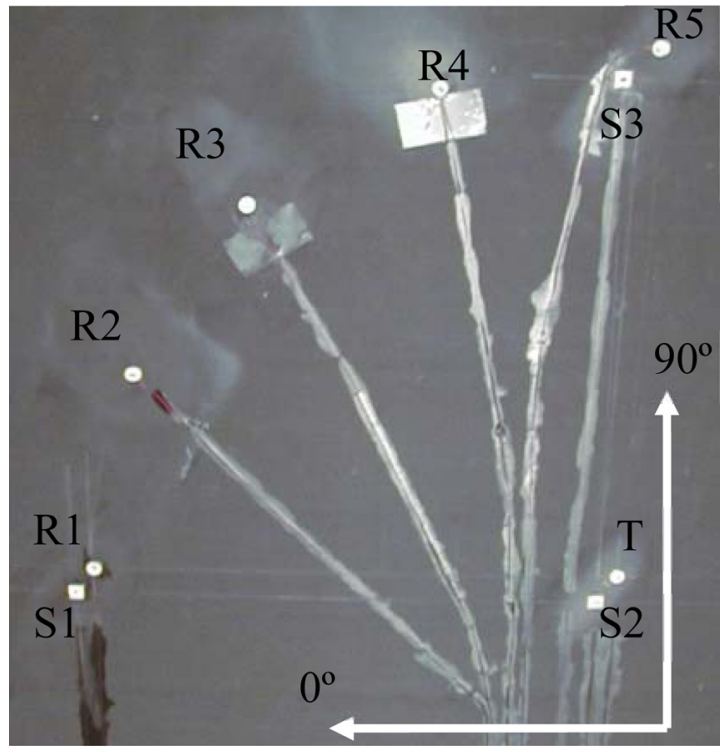

Fig. 3 Experiment setup measuring directional wave speeds in a $[(0 / 45 / 90 /-45) 2] \mathrm{s}$ plate $1240-\mathrm{mm} \times 1240-\mathrm{mm}$ with $2.25-\mathrm{mm}$ thickness. The plate was laminated from T300/5208 unidirectional CFRP tape

to fatigue cracks that propagate under cyclic loading. In contrast, the damage of composite materials occurs in many more ways than that in metals [14], [15]. Composites fail differently under tension than they fail in compression, and the effect of fastener holes is much more complicated than in metals. In addition, the composites are prone to hidden damage from low-velocity impact (e.g., the drop of a hand tool); such damage can be barely visible and may go undetected, but its effect on the degradation of the composite structure strength can be dramatic.

Current design requirements for composite structures are much more stringent than for metallic structures. Military aircraft components have to comply with an Aircraft Structural Integrity Program (ASIP) following the JSSG 2006 [16] and MIL-HDBK-1530 [17] guidelines. Pre-existing manufacturing flaws and serviceinduced cracking are assumed to exist, even if undetected and the ASIP function is to manage this fact while preventing aircraft accidents and downtime. In general, metallic structures are allowed to exhibit a certain amount of subcritical crack growth within the design life of the component. Detectable cracks are noted and managed as part of the maintenance and inspection process. In contrast, no known delamination-cracks are allowed to exist (much less grow) in composite structures. However, the composite components are generally designed to tolerate a certain size of undetectable damage. Of course, this additional "safety margin" comes with a weight penalty, which could be mitigated through better understanding of composite damage detection and management mechanisms.

Composite structures are prone to a particular type of damage that is not critical in metallic aerospace structures, i.e., low-velocity impact damage [18]. Such damage may occur during manufacturing or in service due to, say, a hand tool being dropped onto a thin-wall composite part. A low-velocity impact may damage the structure without leaving any visible marks on the surface ("barely visible damage"). In this case, the impact result takes the form of delaminations in the composite layup. (A more drastic impact may also show spalling on the back side, while having no visible marks on the front side). Delamination due to barely visible impact damage can significantly diminish the composite compression strength (delaminated plies have a much weaker buckling resistance than the same plies solidly bonded together). Manufacturing companies place emphasis on testing the open-hole compression strength after impact as part of the qualifying procedure for their composite structures.

In order to satisfy the damage tolerance requirements, one has to demonstrate that an aircraft structure possesses adequate residual strength at the end of service life in the presence of an assumed worst-case damage, as for example that caused by a lowvelocity impact on a composite structure. This may be accomplished by showing positive margins-of-safety at the maximum recommended load. Worst-case damage is defined as the damage caused by an impact event (e.g., a 1-in. hemispherical impactor) at the lesser of the following two energy levels: (a) $100 \mathrm{ft}-\mathrm{lb}$ or (b) energy to cause a visible dent (0.1-in. deep). Detection of delaminations due to barely visible impact damage is a major emphasis in composite SHM research.

\section{Damage Detection Experiments in Composite Materials}

A series of experiments were performed to detect two types of damage in composite plates. The first type of damage used in these experiments was a small hole of increasing diameter. Holes are generally not a representative type of damage for composite structures; however, we decide to use holes first in our damage detection tests because this type of damage can be easily manufactured and reproduced with accuracy. The second type of damage considered in these experiments was impact damage. This type of damage was produced using an inertial impactor of various weights dropped from various heights.
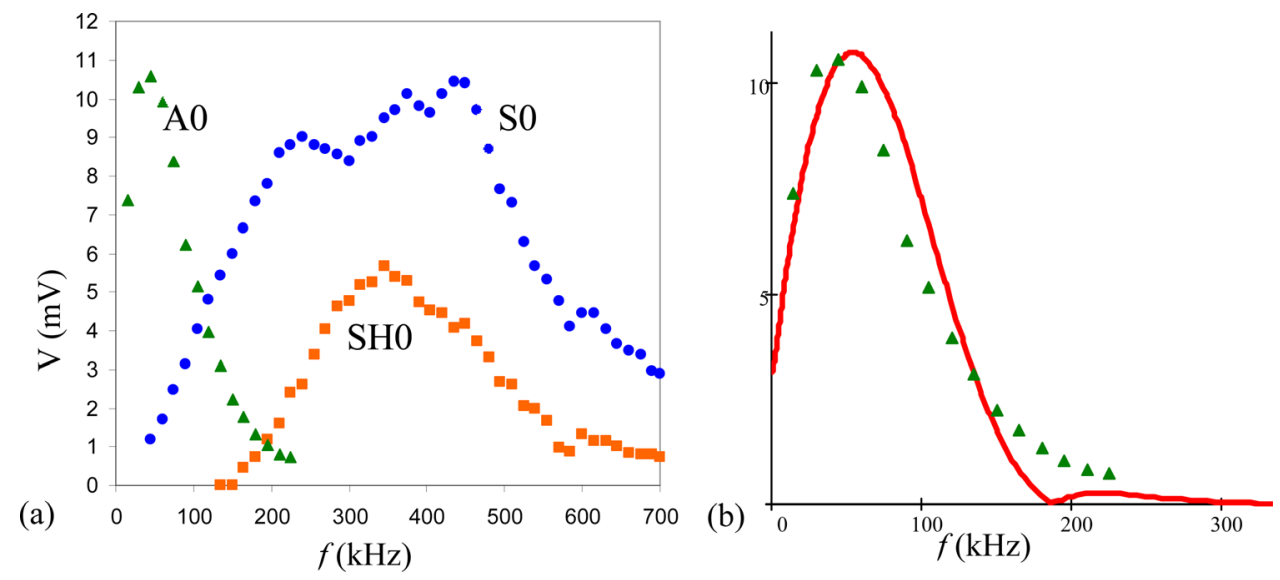

Fig. 4 Tuning of PWAS guided waves in composites: (a) quasi-A0 mode, quasi-S0 mode, quasi-SHO mode. (b) comparison of theoretical prediction (full line) vs. experimental values for A0 mode. 
The specimen consisted of a $1240-\mathrm{mm}$ by $1240-\mathrm{mm}$ quasi-isotropic plate with a $[(0 / 45 / 90 /-45) 2]_{S}$ layup of T300/5208 unidirectional tape; the overall thickness was $2.25 \mathrm{~mm}$. The experimental setup is shown in Fig. 5; the label "hole" indicates the location of the hole damage and the labels " 1 " and " 2 " indicate the impact locations. A set of twelve PWAS transducers were installed in pairs. The PWAS pairs were (p0,p1), (p2,p3), (p4,p5), (p8,p9), (p10,p11), (p12,p13). The distance between the PWAS transducers in each pair was $300 \mathrm{~mm}$. The excitation signal was a 3-count 11-V smoothed tone-burst. The data were collected automatically from PWAS p0, p1, p5, p8, p12, and p13 using an ASCU2 automatic signal collection unit (Fig. 5). Each PWAS was in turn transmitter and receiver. Three frequencies were chosen according to the wave tuning principle: (i) $f=54 \mathrm{kHz}$, when only the A0 mode is present; (ii) $f=225 \mathrm{kHz}$, when only the $\mathrm{S} 0$ mode is present; and (iii) $f=255 \mathrm{kHz}$, when the $\mathrm{S} 0$ mode has maximum amplitude. Four sequential baseline readings were taken with the plate undamaged. Subsequent readings were taken after each damage type was applied to the plate.

Hole Damage Detection in a Quasi-isotropic Composite Plate. A hole of increasing size was drilled between PWAS p1 and p12. The location of the hole was halfway between these two PWAS transducers. The diameter of the hole was increased in 14 steps from zero through $\sim 6 \mathrm{~mm}$ (Table 1 ). At each damage step, several readings were taken. Data processing consisted in comparing each reading with the baseline (reading 00) and calculating the damage index (DI). The DI value was computed with the root mean square deviation (RMSD) algorithm. We analyzed the DI data with statistical software and stated our conclusions to a significance of $99 \%$. Results for $f=54 \mathrm{kHz}$, when only the A0 mode is present, are shown in Fig. 6. Similar results were obtained for the other tuning frequencies. From these experiments it was found that, with $99 \%$ confidence, the minimum detectable hole size was $2.77 \mathrm{~mm}$.

Impact Damage Experiments. Two impact damage levels were produced on the plate by choosing different impactor configurations (i.e., weights and heights). Thus three steps were identified (Table 2), with step 1 being "no damage" and step 3 being "maximum damage". The impactor used for damage location 1 had a total weight of $1391 \mathrm{~g}$ ( $3 \mathrm{lb} 1.1 \mathrm{oz}$.). The first impact had an energy level of $6 \mathrm{ft}-\mathrm{lb}$ and hit the plate at about $3.42 \mathrm{~m} / \mathrm{s}(11.22$

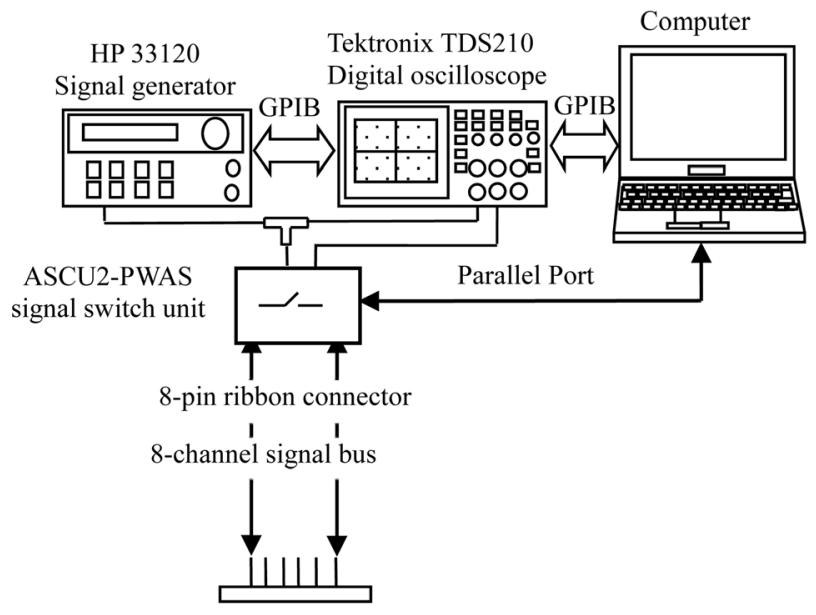

Table 1 Hole diameters for the damage detection experiments on quasi-isotropic composite panel

\begin{tabular}{lccccc}
\hline \hline Step & Reading \# & $\begin{array}{c}\text { Hole size } \\
\text { in mil (mm) }\end{array}$ & Step & Reading \# & $\begin{array}{c}\text { Hole size } \\
\text { in mil (mm) }\end{array}$ \\
\hline 1 & $00-03$ & 0 & 2 & $04-07$ & $032(0.81)$ \\
3 & $08-11$ & $059(1.50)$ & 4 & $12-15$ & $063(1.60)$ \\
5 & $16-19$ & $078(1.98)$ & 6 & $20-23$ & $109(2.77)$ \\
7 & $24-28$ & $125(3.18)$ & 8 & $29-32$ & $141(3.58)$ \\
9 & $33-36$ & $156(3.96)$ & 10 & $37-40$ & $172(4.37)$ \\
11 & $41-44$ & $188(4.78)$ & 12 & $45-48$ & $203(5.16)$ \\
13 & $49-52$ & $219(5.56)$ & 14 & $53-56$ & $234(5.94)$ \\
\hline \hline
\end{tabular}

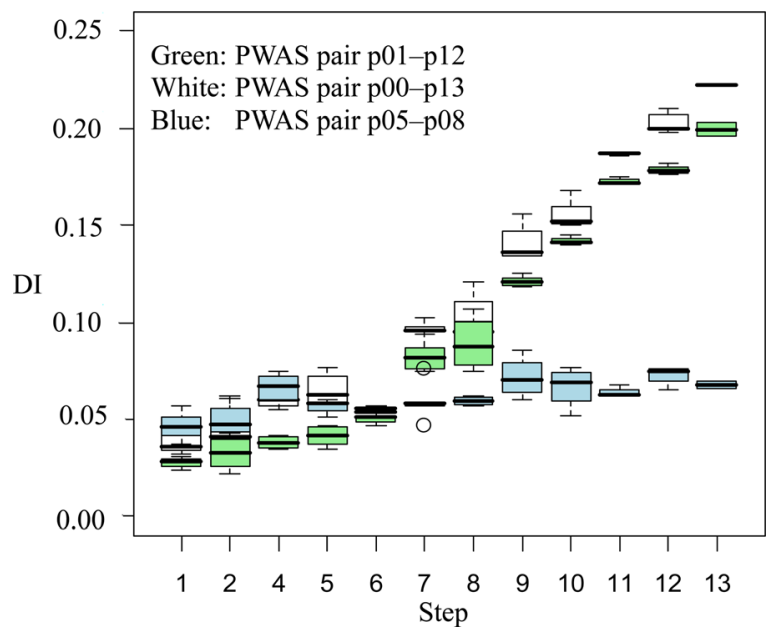

Fig. 6 Pitch-catch hole detection results showing DI values at different damage step values and different PWAS pairs for $f=54 \mathrm{kHz}$, i.e., when only A0 mode is present

Table 2 Synopsis of the impacts applied to the composite plate at location 1

\begin{tabular}{lcccc}
\hline \hline Damage location & Readings & Energy & Velocity & Step \\
\hline 1 & $00-10$ & - & - & 1 \\
& $11-20$ & $6 \mathrm{ft}-\mathrm{lb}$ & $11.2 \mathrm{ft} / \mathrm{s}$ & 2 \\
& $21-30$ & $12 \mathrm{ft}-\mathrm{lb}$ & $16.0 \mathrm{ft} / \mathrm{s}$ & 3 \\
\hline \hline
\end{tabular}

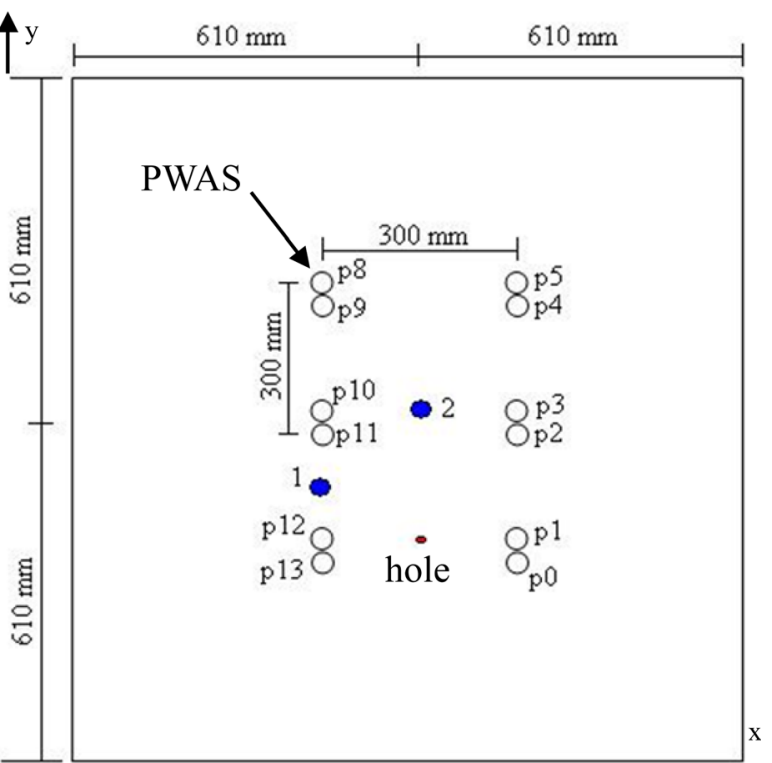

Fig. 5 Experimental set-up for damage detection on quasi-isotropic composite panel: Featured on the plate are: 14 PWAS transducers ( $p 0$ through $p 13$ ); one hole damage; two impact locations (1 and 2) 

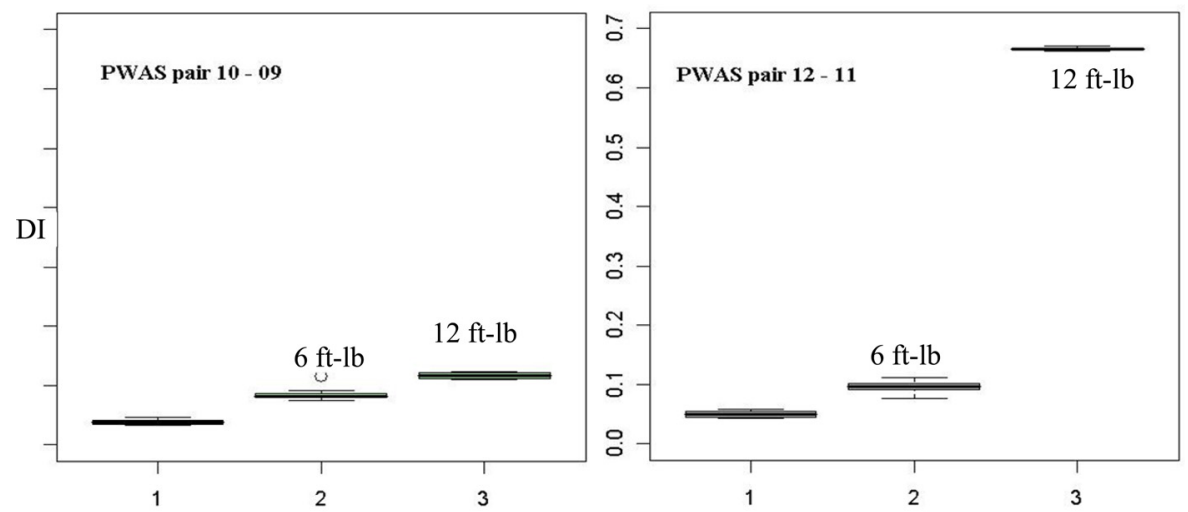

Fig. 7 Detection of impact damage at location 1: DI values as a function of the damage level for PWAS pairs p9-p10 and p11-p12 ( $f=54 \mathrm{kHz}$, i.e., when only A0 mode is present)

$\mathrm{ft} / \mathrm{s}$ ); the second impact had an energy level of $12 \mathrm{ft}-\mathrm{lb}$ and hit the plate at about $4.84 \mathrm{~m} / \mathrm{s}(15.87 \mathrm{ft} / \mathrm{s})$. The impactor used for damage location 2 had a total weight of $891 \mathrm{~g}$ (1 lb $15.5 \mathrm{oz}$.). The first impact had an energy level of $6 \mathrm{ft}-\mathrm{lb}$ and hit the plate at about $4.28 \mathrm{~m} / \mathrm{sec}(14.03 \mathrm{ft} / \mathrm{s})$; the second impact had an energy level of $12 \mathrm{ft}-\mathrm{lb}$ and hit the plate at about $6 \mathrm{~m} / \mathrm{sec}(19.83 \mathrm{ft} / \mathrm{s})$. The detection consisted in sending pitch-catch waves packets at each of the three tuning frequencies: (i) $f=54 \mathrm{kHz}$, when only the A0 mode is present; (ii) $f=225 \mathrm{kHz}$, when only the $\mathrm{S} 0$ mode is present; and (iii) $f=255 \mathrm{kHz}$, when the $\mathrm{S} 0$ mode has maximum amplitude. Figure 7 shows the results for the impact at location 1 and two PWAS pairs, 10-09 and 12-11. It is apparent that the damage is detected. However, the PWAS pair 12-11 shows a higher sensitivity to the detection of the $12 \mathrm{ft}-\mathrm{lb}$ damage than the PWAS pair 10-09. Similar results were obtained for the damage location 2.

\section{Summary and Conclusion}

This paper has presented an investigation in the use of PWAS to perform SHM of composite structures using tuned guided waves. The paper started with an introduction of the PWAS transducers and a review of the various ways in which PWAS may detect damage using traveling guided waves, standing waves, and phased arrays. Next, the paper discussed the challenge of guided wave propagation in composite materials and presented some analytical predictions of the dispersion curves which are more complicated that for isotropic metallic materials. However, it was shown that the wave tuning effect initially identified for metallic materials can be also identified in composite materials; tuning measurements performed on a composite plate were presented in comparison with theoretical predictions.

After discussing briefly the damage modes commonly met in structural composites, the paper presented experimental results obtained on a large composite plate. Two damage types were considered: (i) hole damage; and (ii) impact damage. The detection method used in these experiments was pitch-catch with three tuning frequencies were: (i) $f=54 \mathrm{kHz}$, when only the $\mathrm{A} 0$ mode is present; (ii) $f=225 \mathrm{kHz}$, when only the $\mathrm{S} 0$ mode is present; and (iii) $f=255 \mathrm{kHz}$, when the $\mathrm{S} 0$ mode has maximum amplitude. It was found that, with $99 \%$ confidence, the minimum detectable hole size was $2.77 \mathrm{~mm}$. In the impact damage experiments, it was found that tuned $\mathrm{A} 0$ guided wave mode were much more effective in detecting impact damage in quasi-isotropic carbon-epoxy plates than S0 mode. It was also found that, because of the anisotropy of the composite material, the directional placement of the PWAS transducers plays an important role in their detection capabilities.

However, the results presented in this paper are exploratory in nature and preliminary. Further research needs to be done to better understand the interaction of guided waves with damage in composite materials and how various guide-wave types interact with various types of damage.

\section{References}

[1] Sohn, H., Farrar, C. R., Hemez, F. M., Shunk, D. D., Stinemates, S. W., Nadler, B. R., and Czarnecki, J. J., 2004, "A Review of Structural Health Monitoring Literature form 1996-2001," Los Alamos National Laboratory, Report No. LA13976-MS.

[2] Farrar, C. R., Sohn, H., Hemez, F. M., Anderson, M. C., Bement, M. T., Cornwell, P. J., Doebling, S. W., Schultze, J. F., Lieven, N., and Robertson, A. N. 2004, "Damage Prognosis: Current Status and Future Needs," Los Alamos National Laboratory, Report No. LA-14051-MS.

[3] Rose, J. L., and Soley, L., 2000, "Ultrasonic Guided Waves for the Detection of Anomalies in Aircraft Components," Mater. Eval., 50(9), pp. 1080-1086.

[4] Giurgiutiu, V., 2008, Structural Health Monitoring With Piezoelectric Wafer Active Sensors, Elsevier Academic Press, New York, pp. 239-308.

[5] Soutis, C., and Beaumont, P. W. R., 2005, Multi-Scale Modelling of Composite Material Systems: The Art of Predictive Damage Modelling, Woodhead Publishing Ltd., Cambridge, UK.

[6] Giurgiutiu, V., and Xu, B., 2007, "Self-Processing Integrated Damage Assessment Sensor for Structural Health Monitoring (SPIDAS)," U.S. Patent No. $\# 7,174,255$.

[7] Giurgiutiu, V., 2006, "In-Situ Structural Health Monitoring, Diagnostics, and Prognostics System Utilizing Thin Piezoelectric Sensors," U.S. Patent No. $\# 7,024,315$.

[8] Giurgiutiu, V., Bao, J., Zagrai, A. N., 2006, "Structural Health Monitoring System Utilizing Guided Lamb Waves Embedded Ultrasonic Structural Radar,' U.S. Patent No. \#6,996,480.

[9] Worlton, D. C., 1957, Ultrasonic Testing With Lamb Waves, Nondestr. Test., 15(4), pp. 218-222.

[10] Rose J. L., 1999, Ultrasonic Waves in Solid Media, Cambridge University Press.

[11] Wilcox, P., Lowe, M., and Cawley, P., 2000, "Lamb and SH Wave Transducer Arrays for the Inspection of Large Areas of Thick Plates," Review of Progress in Quantitative Nondestructive Evaluation, Thompson, D. O. and Chimenti, D. E., eds., Plenum Press, New York, pp. 1049-1056.

[12] Diamanti, K., Soutis, C. and Hodgkinson, J. M., 2007, "Piezoelectric Transducer Arrangement for the Inspection of Large Composite Structures," Composites A, 38(4), pp. 1121-1130.

[13] Nayfeh A. H., 2007, Wave Propagation in Layered Anisotropic Media With Application to Composites, Elsevier.

[14] Tong L. and Soutis, C., 2003, Recent Advances in Structural Joints and Repairs for Composites Materials, Kluwer Academic Publishers, The Netherlands.

[15] Diamanti, K., Soutis, C. and Hodgkinson, J. M., 2005, "Non-Destructive Inspection of Sandwich and Repaired Composite Laminated Structures," Compos. Sci. Technol., 65(13), pp. 2059-2067.

[16] JSSG-2006, "Aircraft Structures, General Specification for", US Dept. of Defense Joint Specification Guide JSSG-2006, 30 Oct. 1998, http://www.every spec.com/DoD/DoD+PUBLICATIONS/JSSG-2006_10206/

[17] MIL-HDBK-1530, "Aircraft Structural Integrity Program, General Guidelines for" US Dept. of Defense Military Handbook, 4 Nov. 1996, http://www.every spec.com/MIL-HDBK/MIL-HDBK+(1500+-+1799)/MIL_HDBK_1530_1844/

[18] Heida, J. H., Konijnenberg, P., Hart, W. G. J., 1995, "Characterization of Impact Damage in Carbon-Epoxy Composites," Impact and Dynamic Fracture of Polymers and Composites, Williams, J. G. and Pavan, A., eds., Mech. Eng. Publications, London, ESIS 19, pp. 359-370. 